\title{
Correlative Electron Microscopy and NanoSIMS Analysis for Lipid Studies
}

\author{
Haibo Jiang ${ }^{1,2}$, Cuiwen $\mathrm{He}^{2}$, Loren Fong ${ }^{2}$ and Stephen Young ${ }^{2}$ \\ 1. School of Molecular Sciences, The University of Western Australia, Perth, Australia. \\ 2. Department of Medicine, University of California, Los Angeles, CA.
}

Lipids are essential molecules in organisms. They are critical components of membrane structures and also important sources of energy and carbon, and play critical roles in signalling pathways. Abnormalities in lipid metabolism can also lead to severe health conditions. However, our understanding of lipids is far less than our understanding of proteins. We are at a stage where there are tremendous potential opportunities, requiring a more concentrated effort in lipid research. There are many factors limiting the progressing of lipid studies, and one of the most important ones is the lack of powerful techniques. A few years ago, we started to develop new methods to use correlative nanoscale secondary ion mass spectrometry (NanoSIMS) and electron microscopy to visualize lipids in biological systems [1-3]. Indeed, this approach proved to be powerful. This presentation will discuss two recent studies using the correlative method: (1) how fatty acids products from lipolysis transport through capillary endothelial cells; and (2) the development of the ${ }^{15} \mathrm{~N}$-ALOD4 probe to visualize and quantify cholesterol on cell plasma membrane.

For decades, those interested in intravascular lipolysis have been handicapped by an inability to visualize lipid transport. Lipid transport was studied indirectly by administering radiolabeled lipids to animals, grinding up tissues, measuring lipids, and scintillation counting. These types of experiments have been extended to knockout models. While this approach has yielded key insights, information on subcellular compartments and different cell types is lost. We have applied correlative electron microscopy and NanoSIMS analysis to study the lipid transport through capillary endothelial cells. The quality of NanoSIMS imaging for capillary endothelial cells is high; one can visualize both luminal and abluminal plasma membranes. To aid in the interpretation of NanoSIMS images, we obtain backscattered electron (BSE) from a scanning electron microscope on the very same sections for the ultrastructural information [1] (Figure 1). Using this correlative method, we have (1) studied the mechanisms of the marginations of triglyceride-rich lipoproteins (TRLs) along luminal side of capillary endothelial cells [4]; (2) showed the transport of intact TRLs through capillary endothelial cells; and (3) quantified the delivery of lipids to the heart and BAT of wild-type and Gpihbpl ${ }^{-/}$mice (where lipolysis is defective) [5]. More recently, we visualized the movement of TRL-derived lipids across heart capillaries. Results have shown that the fatty acids products of TRL processing move across capillary endothelial cells very rapidly (within a minute), and endothelial cells may not be a control point for lipid movement into cardiomyocytes. Remarkably, a deficiency of the putative fatty acid transport protein, CD36, which is expressed highly in capillary endothelial cells, did not impede entry of TRL-derived nutrients into cardiomyocytes.

Imaging cholesterol within cells and tissues has been challenging because the behaviour of fluorescently labelled cholesterol analogues behave differently from unmodified cholesterol. Using a similar strategy, we have visualized and quantified stable-isotope labelled cholesterol in cells and tissues. We developed mutants of ${ }^{15} \mathrm{~N}$-labeled cholesterol-binding cytolysins (PFO*, ALO-D4) to visualize the distribution of the "accessible pool" of cholesterol on the plasma membrane of Chinese hamster ovary K1 cells [6]. These studies revealed that "accessible cholesterol" is not evenly distributed on the plasma membrane 
but instead is enriched on the surface of microvilli. The accessible cholesterol on microvilli could be relevant to the movement of cholesterol away from the plasma membrane. This presentation will also discuss the recent developments using correlative electron microscopy and NanoSIMS analysis in our cholesterol transport studies.

References:

[1] H Jiang et al, Methods 68(2) (2014), p. 317.

[2] H Jiang et al, Current Opinion in Biotechnology 41 (2016), p. 130.

[3] C He et al, Journal of Investigative Medicine Oct 28 (2016).

[4] L Fong et al, Trends in Endocrinology \& Metabolism 27(7) (2016), p. 455.

[5] Jiang et al, Journal of Lipid Research 55(10) (2014), p. 2156.

[6] C He et al, Proceedings of the National Academy of Sciences (2017) p. 201621432.

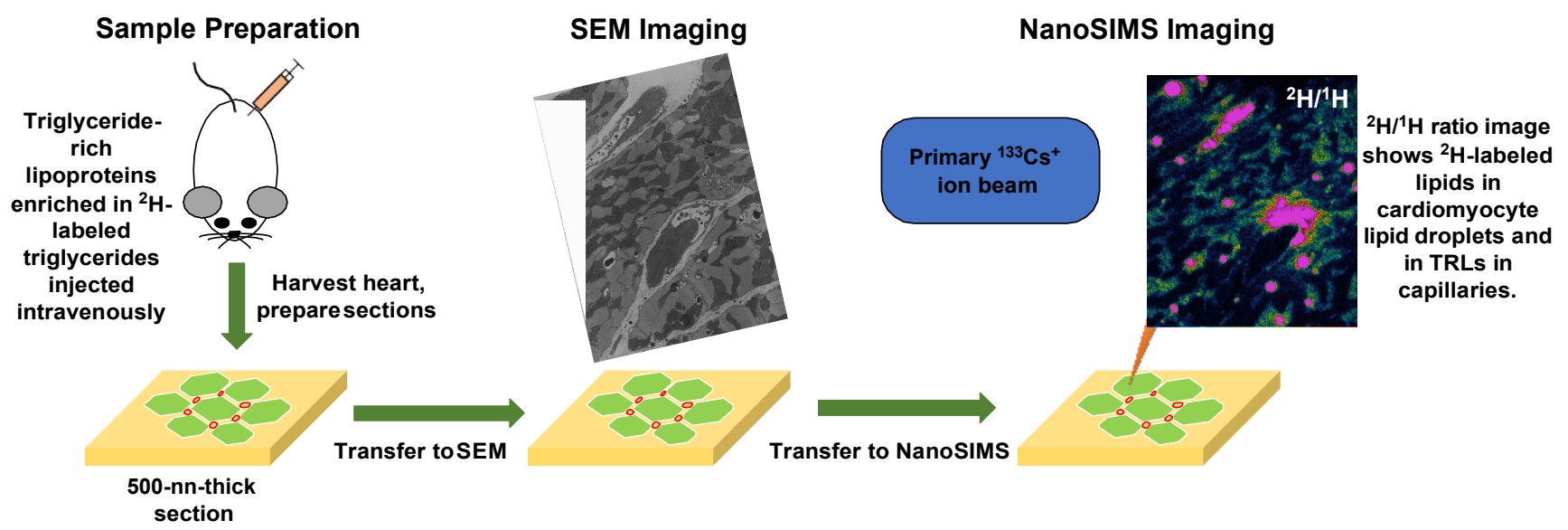

Figure 1. Workflow of the correlative EM and NanoSIMS analysis for lipid studies. 